\title{
Serial counts of Mycobacterium tuberculosis in sputum as surrogate markers of the sterilising activity of rifampicin and pyrazinamide in treating pulmonary tuberculosis Richard Brindle ${ }^{1}$, Joseph Odhiambo ${ }^{2}$ and Denis Mitchison*3
}

Address: 1 Public Health Laboratory, Portsmouth Hospital, Milton Rd, Portsmouth, PO3 6AO, ${ }^{2}$ Kenya Medical Research Institute, P.O. Box 47855, Nairobi, Kenya and 3Department of Medical Microbiology, St George's Hospital Medical School, Cranmer Terrace, London SW17 ORE, UK

E-mail: Richard Brindle - Richard.Brindle@porthosp.nhs.uk; Joseph Odhiambo - JOdhiambo@NAIROB.MIMCOM.NET; Denis Mitchison* - dmitchis@sghms.ac.uk

${ }^{*}$ Corresponding author

This article is available from: http://www.biomedcentral.com/I47I-2466/I/2

(C) 200 I Brindle et al; licensee BioMed Central Ltd. Verbatim copying and redistribution of this article are permitted in any medium for any non-commercial purpose, provided this notice is preserved along with the article's original URL. For commercial use, contact info@biomedcentral.com

\begin{abstract}
Background: Since the sterilising activity of new antituberculosis drugs is difficult to assess by conventional phase III studies, surrogate methods related to eventual relapse rates are required.

Methods: A suitable method is suggested by a retrospective analysis of viable counts of Mycobacterium tuberculosis in 12-hr sputum collections from 122 newly diagnosed patients with pulmonary tuberculosis in Nairobi, done pretreatment and at 2, 7, 14 and 28 days. Treatment was with isoniazid and streptomycin, supplemented with either thiacetazone (SHT) or rifampicin + pyrazinamide (SHRZ).

Results: During days 0-2, a large kill due to isoniazid occurred, unrelated to treatment or HIV status; thereafter it decreased exponentially. SHRZ appeared to have greater sterilising activity than SHT during days $2-7(p=0.044)$, due to rifampicin, and during days $14-28$, probably due mainly to pyrazinamide. The greatest discrimination between SHRZ and SHT treatments was found between regression estimates of kill over days $2-28(p=0.0005)$ in patients who remained positive up to 28 days with homogeneous kill rates. No associations were found between regression estimates and the age, sex, and extent of disease or cavitation. An increased kill in HIV seropositive patients, unrelated to the treatment effect, was evident during days $2-28(p=0.007)$, mainly during days $2-7$.

Conclusions: Surrogate marker studies should either be in small groups treated with monotherapy during days 2 to about 7 or as add-ons or replacements in isoniazid-containing standard regimens from days 2 to 28 in large groups.
\end{abstract}

\section{Background}

The aim of the Global Alliance for Tuberculosis Drug Development, formed during 2000 , is to discover and test new antituberculosis drugs, particularly those that have high sterilising activity and are therefore likely to be able to shorten the duration of treatment [1]. Unfortunately, the phase III clinical trials necessary to establish efficacy in human disease need large numbers of patients, take 
several years to perform, and even then may fail to establish the activity of the new drug, since the action of a single new drug is difficult to detect in multi-drug regimens. The end-point of conventional phase III trials is the relapse rate after treatment. Surrogate markers for relapse are therefore required to obtain a preliminary indication of efficacy and sterilising activity of the drug, even though a phase III trial with estimation of relapse rates is eventually necessary. The best validated surrogate marker for relapse is the proportion of sputum cultures that remain positive after about 2 months of chemotherapy. This has been shown to associate with the fall in relapse rates resulting from the addition of rifampicin and pyrazinamide to regimens of other drugs in 8 clinical trials $[2,3]$. Less well validated procedures are the measurement of the 85B (alpha) antigen of M. tuberculosis during chemotherapy [4] and the frequency of positive cultures at 28 days using the BACTEC system [5]. All of these markers measure the bactericidal activity of drugs during periods of at least 15 days of treatment. Studies of early bactericidal activity (EBA) have usually been carried out over the first 2 days of treatment. They then measure the activity of different drugs and different doses of the same drug against rapidly multiplying extra-cellular M. tuberculosis in cavity walls but do not indicate sterilising activity $[6,7]$. However, a recent multi-centre study explored a period of 5 days from the start of treatment and found that the extended EBA over the period from 2 to 5 days might have measured the high sterilising activity of rifampicin [8].

The possibility of exploring the relative efficiencies of measures of bactericidal activity during various periods of chemotherapy arose from a unique study in Nairobi of counts of viable $M$. tuberculosis in sputum, cultured in the same manner as in EBA studies [9]. These counts were done on 122 patients during the first month of treatment with either streptomycin, thiacetazone and isoniazid (SHT) or with streptomycin, isoniazid, rifampicin and pyrazinamide (SHRZ) given daily. Fortunately, they included counts after 2 days of treatment, the critical time point from which to measure sterilising activity as indicated in the recent EBA study extended over a period of 5 days [8]. A comparison between the bactericidal activities of different periods of treatment with these regimens would measure the effect of an addon to streptomycin/isoniazid $(\mathrm{SH})$ of either thiacetazone, a non-sterilising drug, or of rifampicin and pyrazinamide, the two most active sterilising drugs rifampicin and pyrazinamide [10]. Many of these patients were HIV seropositive, and the HIV status of all was known, so that the influence of HIV serology could also be studied. The main aim of the original report on this study was to examine the effect of HIV status on the response to chemotherapy. The data files from this study were still available and have been re-analysed principally to see what differences could be found between the two treatment regimens in respect of EBA responses during the period of 2-7 days, and of responses over later periods during treatment. Highly significant differences between the bactericidal activities of the two regimens were found during the 2-28 day period suggesting the value of this approach.

\section{Methods \\ Patients}

Consecutive patients were recruited from the Infectious Diseases Hospital, Nairobi, who were 15 yr or older, with newly diagnosed, previously untreated pulmonary TB. All the patients gave informed consent to participation in the study and HIV antibody testing. The study was approved by the Kenya National Ethical Committee. Only those patients who were culture positive with drug-sensitive $M$. tuberculosis before treatment were analysed. These patients were also recruited into a prospective study of HIV and tuberculosis in which a wide range of clinical, laboratory, and epidemiological factors were examined [21].

HIV serology was performed using two enzyme immunoassays (EIA), as described previously [9]. Posteroanterior chest radiographs were divided horizontally by eye into three equal zones per lung field. The extent of disease was determined by the number of zones containing lesions in the lung parenchyma. The number of lung zones with cavities was also recorded. Two treatment regimens were examined sequentially. During the first month, all drugs were given daily, 7 days in the week. Patients given the SHT regimen received HT as isoniazid $300 \mathrm{mg}$ and thiacetazone $150 \mathrm{mg}$, and those given the SHRZ regimen received the HRZ as a combined preparation ("Rifater", Merrel-Dow). Streptomycin $1 \mathrm{~g}$ was given intramuscularly in both regimens.

\section{Bacteriology}

Bacteriological culture was similar to that described in previous EBA studies [8]. Sputum was collected in widemouthed glass jars over a 12-h period, from 8 p.m. to 8 a.m. the next day on Days o, 2, 7, 14, and 28. Therapy was started immediately following collection of the Day-o specimen and thereafter was given daily after 8 a.m. Dosage and sputum collection times were so arranged that patients had received 2 daily doses of treatment before the 2-day time collection, 7 daily doses before the 7-day collection and so on. Middlebrook 7H11 (Difco Code o838), supplemented with oleic acid-albumen-catalase (OADC, Difco Code 0722), was used for the viable counts. It was made selective by the addition of $200 \mathrm{u} / \mathrm{ml}$ of polymixin B, $100 \mathrm{mg} / \mathrm{L}$ of carbenicillin, $10 \mathrm{mg} / \mathrm{L}$ of trimethoprim, and $10 \mathrm{mg} / \mathrm{L}$ of amphotericin B (Mast, Code 
MS24) [22]. The sputum was homogenized by adding glass balls ( $15 \mathrm{~mm}$ diameter) to the glass jar, which was sealed in a plastic bag and shaken mechanically for 10 min. A 1-ml sample of the homogenized sputum was mixed with $2 \mathrm{ml}$ diluted dithiothreitol (Sputasol ${ }^{\circledR}$; Oxoid Code SR89) and then vortex mixed for $30 \mathrm{~s}$. The mixture of sputum and dithiothreitol was allowed to stand for 15 min and again vortex mixed. An inoculum of $0.05 \mathrm{ml}$ from each of a series of five 10-fold dilutions of the sputum mixture was spread onto a one-third segment of a7H11 agar plate. The plates were sealed in polyethylene bags and incubated at $37^{\circ} \mathrm{C}$ for up to $4 \mathrm{wk}$ in air and in darkness. Following incubation, colonies were counted on dilutions yielding between 10 and 100 colonies. The colony counts were analysed as $\log _{10}$ colony forming units (CFU) / ml undiluted sputum.

\section{The kill index (KI) and regression coefficients (b)}

No attempt was made to give specimens that were unavailable or gave a negative culture an arbitrary cfu score since a negative culture could indicate a score anywhere between the value for 1 colony $(1.78 \log \mathrm{cfu} / \mathrm{ml})$ and $\mathrm{mi}-$ nus infinity; the results on these specimens were not used in the anlysis. For each of the intervals between sputum collections, a kill index (KI) was calculated as the decrease in the count of colony forming units (cfu) per day between the counts at the two time points indicated in parenthesis, without using counts at intermediate time points. Thus, for the sputum collections over the first two days of treatment KI (O-2) $=\left(\log _{10} \mathrm{cfu} / \mathrm{ml}\right.$ pretreatment $-\log _{10} \mathrm{cfu} / \mathrm{ml}$ at 2 days)/ 2 and during the interval between day-2 and day-28, KI (2-28) $=\left(\log _{10} \mathrm{cfu} /\right.$ $\mathrm{ml}$ at 2 days $-\log _{10} \mathrm{cfu} / \mathrm{ml}$ at 28 days)/26. A KI was calculated for each of the 10 possible time intervals. Coefficients of the linear regression of $\log _{10} \mathrm{cfu} / \mathrm{ml}$ sputum on days of treatment (b) were calculated when counts were available at more than two time points, for instance, on the counts at 2, 7 and 14 days.

\section{Statistical analysis}

The results were analysed in EpiInfo, version 6.o (Centers for Disease Control, Atlanta, GA) for distributions, calculation of individual regression coefficients (b) for each patient and multiple regression analyses, and in STATA, release 6.o (College Station, Texas, TX) for Shapiro-Wilk tests for normality [23], t-tests with unequal variances and 2-way analyses of variance (anova) on unbalanced blocks. In calculating means and SDs, the sign of $b$ was reversed to make comparisons with the KI values easier. Since the values of KI (O-2), KI (O-7) and b (2 onwards) departed considerably from normality in the Shapiro-Wilk tests with skewed means and high kurtosis, the values of KI (0-2) and KI (2-7) were normalised for anova using the mean symmetry version of the BoxCox transformation [24]. Values of b (2 onwards) were normalised by transformation to $1 /(1-b)^{2}$, in which $1-b$ avoided a mix of positive and negative values but left the anova unaltered, while the remaining part of the transformation was chosen using the empirical approach of the ladder command in STATA [25]. All transformed values and the remaining KIs with different ranges were found not to depart significantly from normality by the Shapiro-Wilk test.

\section{Results}

The study was done between April 1989 and December 1990. There were 122 patients with cfu counts values at any time available, but the numbers of patients with counts at each sampling time decreased by 33 (27\%) of the 122 at 2 days and finally by $54(44 \%)$ at the 28 -day period (Table 1). In some instances, sputum gave a nega-

Table I: Patients with sputum specimens not available or negative on culture

Duration of treatment (days) Patients $(n=122)$

Not available

No.
$\%$
Culture negative

No.
$\%$

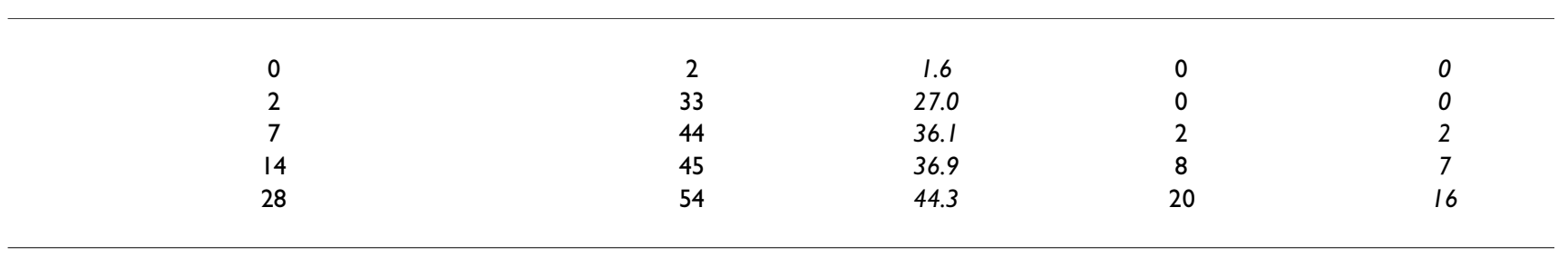


tive culture, but in many instances, the result was unavailable for a variety of reasons that could not be identified, including failure to cough up sputum, drying up of the specimen, breakage of sputum containers and failure of compliance by the patient. Positive HIV serology was found in 17 (25\%) of 67 patients treated with SHT and in $20(36 \%)$ of 55 treated with SHRZ. The incidence of negative and unavailable cultures was fairly similar in all of the four subgroups of patients. A comparison of the characteristics of the patients in the SHT series and the SHRZ series (Table 2) shows no statistically significant differences in their age, sex or initial severity of disease. A similar comparison (Table 2) between HIV seronegative and seropositive patients, shows fewer lung zones involved, less cavitation and lower sputum cfu counts in those who were seropositive, as previously reported [9].

Table 2: Pretreatment characteristics of patients according to treatment series and HIV status

\begin{tabular}{lcccc}
\hline & SHT & SHRZ & HIV- & HIV+ \\
\cline { 2 - 5 } & & & & \\
No. of patients & 67 & 55 & 85 & 37 \\
Mean age & 30.0 & 30.3 & 29.5 & 31.5 \\
Per cent males & 59.7 & 60.0 & 61.2 & 56.8 \\
Mean lung zone (Max. 6) & 4.37 & 4.15 & 4.48 & $3.78^{*}$ \\
Mean cavitation (Max. 6) & 1.31 & 1.29 & 1.65 & $0.51 \dagger$ \\
Mean initial log cfu count & 6.84 & 6.72 & 6.95 & 6.34 ฯ \\
& & & &
\end{tabular}

${ }^{*} p=0.007 \dagger_{p}=0.000001 \pi_{p}=0.007$

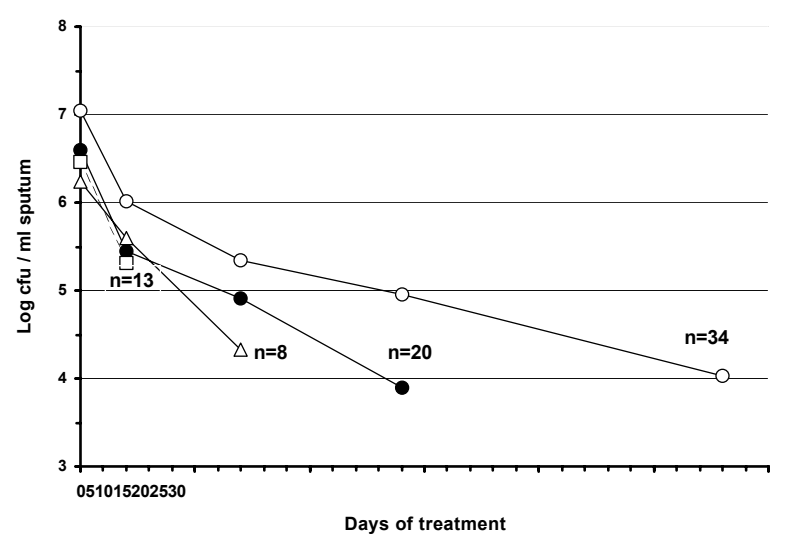

Figure I

Mean cfu counts during chemotherapy in patients who had complete sets of counts up to 2 days of treatment, or up to 7 days or 14 days or 28 days of treatment, but in each case no further positive sputum cultures thereafter.

\section{Regression analyses}

Two measures of the rate of kill, KI and b (with sign reversed), have been used for clarity in nomenclature, but both measure the same daily rate of fall of cfu counts. Each KI is based solely on the two cfu counts indicated in brackets, while $\mathrm{b}$ is based on cfu counts at more than two points times. The cfu counts on sputum from groups of patients, each of whom had a complete set of results over the period that they remained positive are set out in Fig 1. The results from the 34 patients with counts at $0,2,7$, 14 and 28 days are plotted separately from those of the 20 patients with counts at $0,2,7$ and 14 days but with unavailable or negative cultures at 28 days, and so on. In all groups there was an initial abrupt fall in counts of about $1 \log$ unit during the first 2 days. Thereafter, from 2 days onwards, the counts fell more slowly and appeared linearly related to $\log$ cfu. However, the slope of the fitted lines appeared to be steeper when counts became unavailable or negative early in treatment. Mean values of $b$ \pm SD for all available patients were $0.263 \pm 0.245$ for the 8 patients in the 7-day group, $0.165 \pm 0.099$ for the 24 patients in the 14-day group and $0.077 \pm 0.049$ for the 41 patients in the 28-day group. The difference between the mean values of $b$ for the 7-day and 14-day groups is not significant but, between the 14-day and the 28-day groups, it is highly significant (t test with unequal variances; $p=0.0002$ ). Since the rates of sputum conversion of the 14-day and 28-day groups were different, we explored the cfu counts graphically during treatment in the "pure" 28-day population. A comparison of the mean cfu counts during treatment with SHT and SHRZ suggests that the greatest difference in the slopes occurs during the 2-7 day interval, not during 7-14 days, and then again in the 14-28 day interval (Fig. 2). A similar comparison of the graphs according to HIV status also shows that the greater tendency for counts to fall in the seropositive group occurs during days $2-7$, while the curves remain parallel thereafter (Fig. 3). In both Figs 2 and 3, the data points in each curve fall in an approximately linear manner from day-2 onwards.

The steep fall in cfu counts over the first 2 days of treatment was calculated as the KI $(\mathrm{O}-2)$ in Table 3 . The mean fall in counts was 0.513 per day; no significant differences were found due either to the difference between SHT and SHRZ or the difference in HIV status. None of the contrasts due to treatment or to HIV status attained statistical significance with any value of the KI starting from the pretreatment count, that is $\mathrm{KI}(0-7)$, KI (o-14) or KI $(\mathrm{O}-28)$. The fitting of linear regression lines from day-O was not appropriate because of the high values of $\mathrm{KI}$ (O2).

The regression coefficients for straight lines from 2 to 28 days were then calculated for each patient's data. For the 


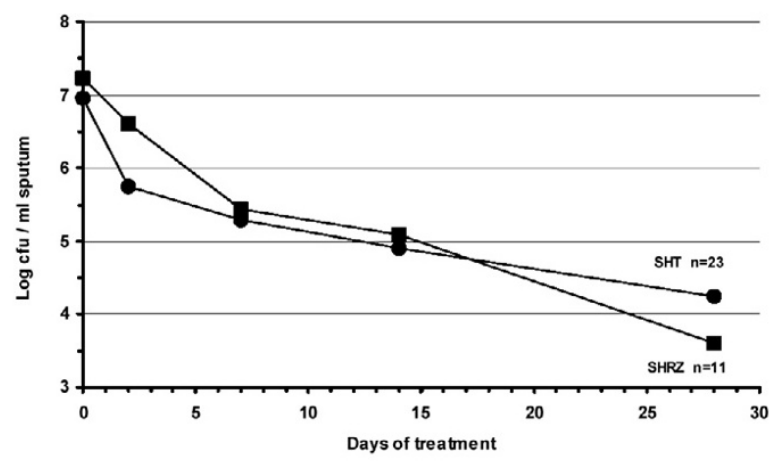

Figure 2

Mean cfu counts during treatment with the STH and the SHRZ regimens in patients who had a complete set of sputum cfu counts up to and including 28 days.

calculation of b ( 2 onwards) in Table 3 , all values of $b$ were analysed including those with counts available at 28 days, or only up to 7 or 14 days; some had missing intermediate counts but were included. When examined by 2-way anova, the difference between the means of $b$ of 0.095 for the 44 SHT patients and 0.163 for the 31 SHRZ patients was highly significant $(\mathrm{p}=0.004)$ as was the difference between the mean of 0.099 for the 52 seronegative patients and 0.177 for the 23 seropositive patients $(p$ $=0.007)$. However, values of this $\mathrm{b}$ contained a high proportion of data from patients who only had positive cultures in the early weeks of treatment and therefore had a fairly high SD (0.116). Further analyses were then done to look at the treatment and HIV effects during time periods of various durations. The KI (2-7) also showed more rapid killing by the SHRZ than the SHT regimens and also more rapid killing by seropositive than by seronegative patients, but the differences for both contrasts bordered on significance $(\mathrm{p}=0.044$ and $\mathrm{p}=0.051$, respectively). Further analyses of KI values were done for the later time intervals. The means for KI (2-7), KI (714) and KI (14-28) fell successively from 0.220 to 0.071 , as did their SDs. In KI (7-14) the means for treatment and HIV status were similar and neither contrast attained significance. However, in the KI (14-18) results, the treatment mean for SHT patients was 0.053, half that of 0.099 for SHRZ patients, and the contrast was suggestive $(\mathrm{p}=0.1)$. Correlation between values of the KI $(2-7)$ and the KI (14-28) in 35 patients gave $r=0.34$, a nonsignificant association ( $\mathrm{p}=0.1-0.05)$.

Finally, the time periods were extended to the $2-28$ day interval. The $b(2-28)$ was calculated on patients all of whom had 28-day counts; there were 35 patients with counts at 2, 7, 14 and 28 days, 4 patients with missing 7day counts and 5 patients with missing 14-day counts. In

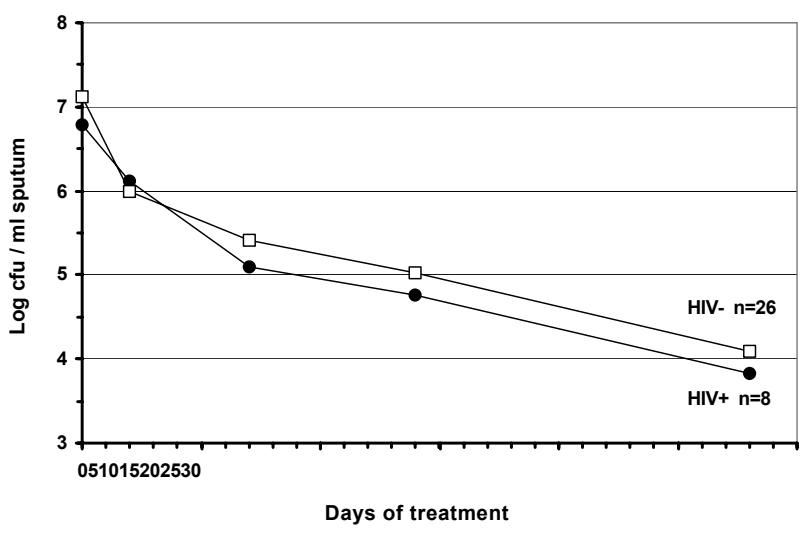

Figure 3

Mean cfu counts during treatment in HIV-negative and HIVpositive patients who had a complete set of sputum cfu counts up to and including 28 days.

both the $\mathrm{b}(2-28)$ and the $\mathrm{KI}(2-28)$ results, the mean decrease in the SHT regimen was about half that in the SHRZ regimen, and the SDs of 0.048 and 0.054 are similar and lower than those encountered in any earlier analysis. The treatment effects of more rapid killing by SHRZ than by SHT in both distributions are highly significant, with higher values of the variance ratio $F$ of 14.4 for the b (2-28) distribution than of 9.9 for the KI (228). No interaction was found between the treatment and HIV status effects in any 2-way anova.

\section{Sputum conversion at $\mathbf{2 8}$ days}

In view of the established value of sputum conversion at 2-months as a predictor of the increase in sterilising activity of a regimen by addition of rifampicin and pyrazinamide, the value of sputum conversion at 28 days was examined in Table 4. Considering only those specimens which gave either a negative or positive culture result, a positive culture was obtained from $29(81 \%)$ of 36 SHT patients and from $19(59 \%)$ of 32 SHRZ patients $(\mathrm{p}=0.1)$ and also from $38(78 \%)$ of 49 HIV seronegative patients and from $10(53 \%)$ of 37 HIV seropositive patients ( $p=$ 0.08).

\section{Influence of patient characteristics on regression}

Multiple regression analyses were carried out relating age, sex, number of lung zones, number of cavities and pretreatment cfu count to the KI (o-2), KI $(2-7)$ and $b(2-28)$. In general no significant associations were found. However, there was a significant regression between the pretreatment counts and $\mathrm{KI}(\mathrm{O}-2)$, and another between the 2-day counts and $\mathrm{KI}(\mathrm{O}-2)$ in the opposite direction, arising because they are not independent. 
Table 3: Summary statistics and main effects of treatment or HIV status in selected distributions

\begin{tabular}{|c|c|c|c|c|c|c|c|}
\hline Variable \& range (days) & $\mathrm{KI}(0-2)$ & b (2 onwards) & $\mathrm{KI}(2-7)$ & $\mathrm{KI}(7-14)$ & KI (14-28) & b (2-28) & KI (2-28) \\
\hline Mean* (\& no. of patients) & $0.513(87)$ & $0.123(75)$ & $0.161(68)$ & $0.094(60)$ & $0.07 \mid(43)$ & $0.077(44)$ & $0.081(44)$ \\
\hline Standard deviation* & 0.554 & 0.116 & 0.220 & 0.123 & 0.072 & 0.048 & 0.054 \\
\hline \multicolumn{8}{|l|}{ Mean* (\& no. of patients): } \\
\hline SHT & $0.540(49)$ & $0.095(44)$ & $0.121(42)$ & 0.091 (38) & $0.053(26)$ & $0.057(27)$ & $0.060(27)$ \\
\hline SHRZ & $0.479(38)$ & $0.163(31)$ & $0.225(26)$ & $0.099(22)$ & $0.099(17)$ & $0.109(17)$ & $0.115(17)$ \\
\hline HIV- & $0.526(59)$ & $0.099(52)$ & $0.124(48)$ & $0.086(44)$ & $0.072(34)$ & $0.076(34)$ & $0.079(34)$ \\
\hline $\mathrm{HIV+}$ & $0.486(28)$ & $0.177(23)$ & $0.250(20)$ & $0.114(16)$ & $0.066(9)$ & $0.080(10)$ & $0.087(10)$ \\
\hline \multicolumn{8}{|l|}{ 2-way anova ${ }^{\dagger}$} \\
\hline Treatment F (\& p) & $0.3(0.6)$ & $8.8(0.004)$ & $4.2(0.044)$ & $0.0(1.0)$ & $2.7(0.1)$ & $14.4(0.0005)$ & $9.9(0.003)$ \\
\hline HIV status F (\& p) & $0.01(0.9)$ & $7.7(0.007)$ & $4.1(0.051)$ & $0.4(0.5)$ & $0.1(0.7)$ & $0.01(0.9)$ & $0.20(0.7)$ \\
\hline
\end{tabular}

\footnotetext{
* Means and standard deviations of untransformed data. † Transformed data for KI (0-2), KI (2-7) and for all values of b except b(2-I4). All interactions between treatment and HIV status were not significant. Statistically significant main effects $(p<0.05)$ are in bold type.
}

\section{Discussion}

The first important finding was the occurrence of a high kill during the first 2 days of treatment shown by the KI (o-2) mean of 0.513 . This was probably due to the daily $300 \mathrm{mg}$ isoniazid in both regimens, since isoniazid has a considerably higher EBA over the first 2 days than other drugs.[6,7] This mean is only a little lower than the mean of 0.575 (95\% Confidence limits $=0.515-0.636$ ) in pooled results obtained with $300 \mathrm{mg}$ isoniazid daily at Stellenbosch University, Cape Town [11]. The SD was however 0.554, considerably higher than the SD of 0.214 in the Cape Town data. These discrepancies might be due to a few patients having had undetected medication with isoniazid before admission to the study, which would result in a low and variable $\mathrm{KI}(\mathrm{O}-2)$. A negative $\mathrm{KI}$ was found in 9 of 87 patients, of whom 7 had a value of $<-0.2$. No urine tests for isoniazid metabolites were done on admission. It is clear that the high and variable kill over the first two days swamps any differences between the sterilising effects of the two regimens and any due to HIV status, and renders useless any regression estimate starting at day-o.

From day-2 onwards, the 2-7 day period showed just significant effects in the treatment comparison. The failure to obtain more clear cut differences, despite being based on 68 patients, is due to the high SD ( \pm 0.22$)$ of KI $(2-7)$, which seems to arise from heterogeneity of kill rates early in treatment (Fig 1). Since heterogeneity is a major factor, only a moderate increase in precision can be expected should more precise estimates of kill rates be obtained by doing counts at intervals during the 2-7 day period. In contrast, substantial differences have been found over the 2-5 day period in comparisons of monotherapy with isoniazid or rifampicin in EBA studies, even though much smaller groups of about 10 patients were examined [8]. This discrepancy suggests that it will be more difficult to establish the sterilising activity of a new drug in the early days of treatment by adding it to an isoniazid-containing regimen than by testing it in monotherapy.

Between 2 and 7 days, it is likely that some phenotypically sensitive organisms occasionally had spurts of metabolism and so were killed preferentially by rifampicin in the SHRZ group [12]. Thereafter between 7 and 14 days, the differential between the means for two regimens disappeared perhaps because the original population had been largely replaced by organisms that are phenotypically resistant [13]. From 14 to 28 days it seems probable that killing started again. This biphasic killing seems to be on different patient populations, because of the poor correlation between the KI (2-7) and the KI (14-28). We suggest that this second phase is due mainly to the sterilising activity of pyrazinamide, for two reasons: (1) pyrazinamide in the mouse starts by being less bactericidal than other drugs, such as isoniazid and streptomycin, but continues at much the same slow rate after the kill by other drugs has almost stopped (Fig 4) [14]. A cross-over point occurs at about 25 days after the start of treatment when the kill due to pyrazinamide begins to predominate. (2) in pulmonary tuberculosis, monotherapy with pyrazinamide has no apparent bactericidal activity during the first 2 days $[6,15]$ and thereafter has slow bactericidal activity up to 14 days with a reduction of sputum cfu counts of only about $1.6 \log$ units $[6,16]$. Presumably this kill continues after 14 days in human disease, as in the mouse, without the slowing of the rate of kill found with other drugs. As shown in clinical studies, the kill due to pyrazinamide must be substantial [10] and must terminate by 8 weeks $[17,18]$; presumably it therefore occurs somewhere between 14 and 56 days. 
Table 4: Sputum conversion at 28 days.

\begin{tabular}{lcccc}
\hline & SHT & SHRZ & HIV- & HIV+ \\
& & & & \\
Not available & 31 & 23 & 36 & 18 \\
Sputum culture: negative & 7 & 13 & 11 & 9 \\
positive & 29 & 19 & 38 & 10 \\
Total patients & 67 & 55 & 85 & 37 \\
& & & & \\
\hline
\end{tabular}

In both the KI ( $0-28)$ values based only on the 2-day and 28-day counts and in the b (2-28) values based on all counts in patients with positive 28 -day cultures, highly significant differences for the treatment effect are evident, with greater killing by the SHRZ regimen. The b $(2-28)$ values are more efficient than those of the KI (228 ), but the increase in efficiency is not that large in view of the greater amount of work that is necessary to obtain them. The high efficiency of the 2-28 day estimates appears due partly to the long time period, but mainly to improved homogeneity of kill rates, as those patients who converted early and also contributed to high early SDs were lost from analysis. These findings support the view, derived from associations between 2-month culture positivity and the addition of sterilising drugs $[2,3]$, that sterilising activity is best shown in a small proportion of patients who convert late.

The high efficiency obtained with regression techniques is to be compared to the failure to show any clear effect of the treatment contrast or the HIV status contrast on the proportions of patients with positive cultures at 28 days. The failure is to be expected since the results of negative/positive culture contrasts at 1-month in clinical trials were much less efficient than those at 2-months in detecting the addition of sterilising drugs [3], and the number of patients, 86 in all, was too small.

The effects of HIV status had a different time course to the treatment effects. HIV status had no effect during the first 2 days but a just detectable effect during days 2-7, with a higher kill in those that were HIV seropositive. That the HIV effect only occurs early is also shown by the high significance $(p=0.007)$ of the HIV contrast in values of $b$ (2 onwards), which includes early converters, but by the non-significance of the b (2-28) or the KI (228 ) contrasts, which do not include early converters. The effect is small probably because the great majority of bacilli are extra-cellular in cavities, well away from professional immune cells $[19,20]$. Since there was no interaction between the HIV status and treatment effects, further studies of determinants of the immune response during the first 2 days of therapy with isoniazid-

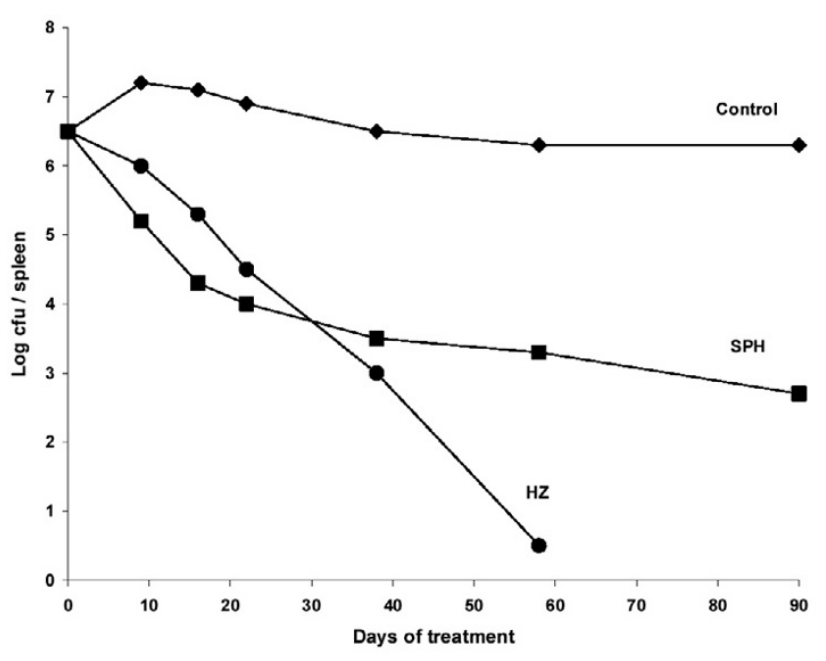

Figure 4

The bactericidal actions of pyrazinamide/isoniazid $(\mathrm{HZ})$ and streptomycin, PAS and isoniazid (SPH) in the spleens of mice. Redrawn from Text-fig. 4 in reference 26.

containing regimens, and even in the succeeding week, are unlikely to yield results helpful to the study of drugaction. Furthermore, there is no reason to exclude HIVpositive patients, or even to identify them, in studies of EBA or in longer-term studies of sterilising activity based on cfu counting in sputum.

In conclusion, these results suggest that identification of the sterilising activity of new drugs could most profitably be done either by EBA studies with monotherapy extending over, at most, the period of 2-9 days after the start of treatment with about 12 patients in each treatment group. It seems unlikely that they could detect differences by adding a new drug to regimens containing isoniazid, nor would they detect sterilising activity similar to that of pyrazinamide. The alternative would be to study add-on or replacement regimens by serial cfu counts on sputum collections starting at day-2 and continuing to day-28 and probably beyond to day-56, in groups of 60 patients or more.

\section{Conclusions}

1. A rapid fall in cfu counts occurred during the first 2 days of treatment, probably due mainly to isoniazid. Thereafter cfu counts fell approximately exponentially.

2. The fall in cfu counts over days $2-7$ was slightly greater in the SHRZ than in the SHT regimen. This difference was probably due to rifampicin in view of the low the bactericidal activity of pyrazinamide at the time. 
3. Calculations of the daily fall in cfu counts over days 228 was a powerful method of demonstrating the long term bactericidal (sterilising) activities of rifampicin and pyrazinamide in the SHRZ regimen.

4. No relation was found between clinical measures of pretreatment severity of disease and the assessments of sterilising activity.

5. Bactericidal activity during the early period from 2 days onwards was greater in HIV seropositive than in seronegative patients but this did not affect the difference between the sterilising activities of the SHT and SHRZ regimens.

\section{Competing interests}

None

\section{Acknowledgement}

We are grateful to the Director of the Kenya Medical Research Institute for his help, and to the staff at its Respiratory Diseases Research Unit who carried out much of the original work on the project, particularly $\mathrm{W}$. Githui, S. Gathua, and P. Waiyaki.

\section{References}

I. Tuberculosis Scientific Blueprint for TB Drug Development. Tuberculosis 200I, 8 I (Suppl.I): I-52

2. Mitchison DA: Assessment of new sterilizing drugs for treating pulmonary tuberculosis by culture at $\mathbf{2}$ months. Am Rev Respir Dis 1993, 147:1062-1063

3. Mitchison DA: Modern methods for assessing the drugs used in the chemotherapy of mycobacterial disease. J Appl Bacteriol 1996, $81: 72 \mathrm{~S}-80 \mathrm{~S}$

4. Wallis RS, Perkins MD, Phillips M, Joloba M, Namale A, Johnson JL, Whalen CC, Teixeira L, Demchuk B, Dietze R, et al: Predicting the outcome of therapy for pulmonary tuberculosis. Am J Respir Crit Care Med 2000, 161:1076-1080

5. Epstein MD, Schluger NW, Davidow AL, Bond SB, Rom WN, Hanna $B$ : Time to detection of $M$. tuberculosis in sputum culture correlates with outcome in patients receiving treatment for pulmonary tuberculosis. Chest 1997, I 1 3:379-386

6. Jindani A, Aber VR, Edwards EA, Mitchison DA: The early bactericidal activity of drugs in patients with pulmonary tuberculosis. Am Rev Respir Dis 1980, 121:939-949

7. Mitchison DA, Sturm WA: The measurement of early bactericidal activity. In: Bailliere's Clinical Infectious Diseases: Mycobacterial Diseases Part II. (Edited by Malin A, McAdam KPWJ) London, Bailliere Tindall $1997185-206$

8. Sirgel FA, Donald PR, Odhiambo J, Githui W, Umapathy KC, Paramasivan CN, Tam CM, Lam KM, Lam CW, Sole KM, Mitchison DA, and the EBA Collaborative Study Group: A multicentre study of the early bactericidal activity of anti-tuberculosis drugs. J Antimicrob Chemother 2000, 45:859-870

9. Brindle RJ, Nunn PP, Githui W, Allen BW, Gathua S, Waiyaki P: Quantitative bacillary response to treatment in HIV-associated pulmonary tuberculosis. Am Rev Respir Dis 1993, 147:958961

10. East African/British Medical Research Council: Controlled clinical trial of four short-course (6-month) regimens of chemotherapy for treatment of pulmonary tuberculosis. Third report. Lancet 1974, 2:237-240

II. Sirgel F, Venter A, Mitchison D: Sources of variation in studies of the early bactericidal activity of antituberculosis drugs. J Antimicrob Chemother 2001, 47:177-182

12. Dickinson JM, Mitchison DA: Experimental models to explain the high sterilizing activity of rifampicin in the chemotherapy of tuberculosis. Am Rev Respir Dis |98I, | 23:367-37|

13. Hu Y, Mangan JA, Dhillon J, Sole KM, Mitchison DA, Butcher PD, Coates ARM: Detection of mRNA transcripts and active tran- scription in persistent Mycobacterium tuberculosis induced by exposure to rifampicin or pyrazinamide. J Bacteriol 2000, I 82:6358-6365

14. McCune RM, Tompsett R, McDermott W: The fate of Mycobacterium tuberculosis in mouse tissues as determined by the microbial enumeration technique. J Exp Med 1956, 104:763-803

15. Botha FJH, Sirgel FA, Parkin DP, van de Wal BW, Donald PR, Mitchison DA: Early bactericidal activity of ethambutol, pyrazinamide and the fixed combination of isoniazid, rifampicin and pyrazinamide (Rifater) in patients with pulmonary tuberculosis. S Afr Med J 1996, 86:155-158

16. Mitchison DA: The action of antituberculosis drugs in shortcourse chemotherapy. Tubercle 1985, 66:219-225

17. East and Central African/British Medical Research Council (fifth Collaborative Study): Controlled clinical trial of 4 short-course regimens of chemotherapy (three 6-month and one 8-month) for pulmonary tuberculosis. Final report. Tubercle 1986, 67:515

18. Hong Kong Chest Service/British Medical Research Council: Controlled trial of 2, 4 and 6 months of pyrazinamide in 6-month, $3 \times$ weekly regimens for smear-positive pulmonary tuberculosis, including an assessment of a combined preparation of isoniazid, rifampicin and pyrazinamide. Am Rev Respir Dis I991, 143:700-706

19. Mitchison DA: The Garrod Lecture. Understanding the chemotherapy of tuberculosis-current problems. J Antimicrob Chemother 1992, 29:477-493

20. Canetti G: The tubercle bacillus in the pulmonary lesion of man: histobacteriology and its bearing on the therapy of pulmonary tuberculosis. New York, Springer 1955

21. Nunn P, Brindle R, Carpenter L, Odhiambo J, Wasunna K, Newnham R, Githui W, Gathua S, Omwega M, McAdam K: Cohort study of human immunodeficiency virus infection in patients with tuberculosis in Nairobi, Kenya. Analysis of early (6 month) mortality. Am Rev Respir Dis 1992, 146:849-854

22. Mitchison DA, Allen BW, Carrol L, Dickinson JM, Aber VR: A selective oleic acid albumin agar medium for tubercle bacilli. J Med Microbiol 1972, 5:165-175

23. Royston $\mathrm{P}: \mathrm{A}$ toolkit for testing for non-normality in complete and censored samples. Statistician 1993, 42:37-43

24. Box GEP, Cox DR: An analysis of transformations. J R Stat Soc 1964, 26:2II-243

25. Tukey JW: Exploratory Data Analysis. Reading, MA. Addison-Wesley Publishing Company 1977

26. McCune RM, Tompsett R, McDermott W: The fate of Mycobacterium tuberculosis in mouse tissues as determined by the microbial enumeration technique. II. The conversion of tuberculous infection to the latent state by the administration of pyrazinamide and a companion drug. J Exp Med 1956, 104:763-803

Publish with BioMed Central and every scientist can read your work free of charge

"BioMedcentral will be the most significant development for disseminating the results of biomedical research in our lifetime." Paul Nurse, Director-General, Imperial Cancer Research Fund

Publish with BMC and your research papers will be:

- available free of charge to the entire biomedical community

- peer reviewed and published immediately upon acceptance

- cited in PubMed and archived on PubMed Central

- yours - you keep the copyright 